Document downloaded from:

http://hdl.handle.net/10251/143127

This paper must be cited as:

Gregori Gregori, V.; Miñana, J.; Miravet-Fortuño, D. (12-2). Fuzzy Partial Metric Spaces. International Journal of General Systems. https://doi.org/10.1080/03081079.2018.1552687

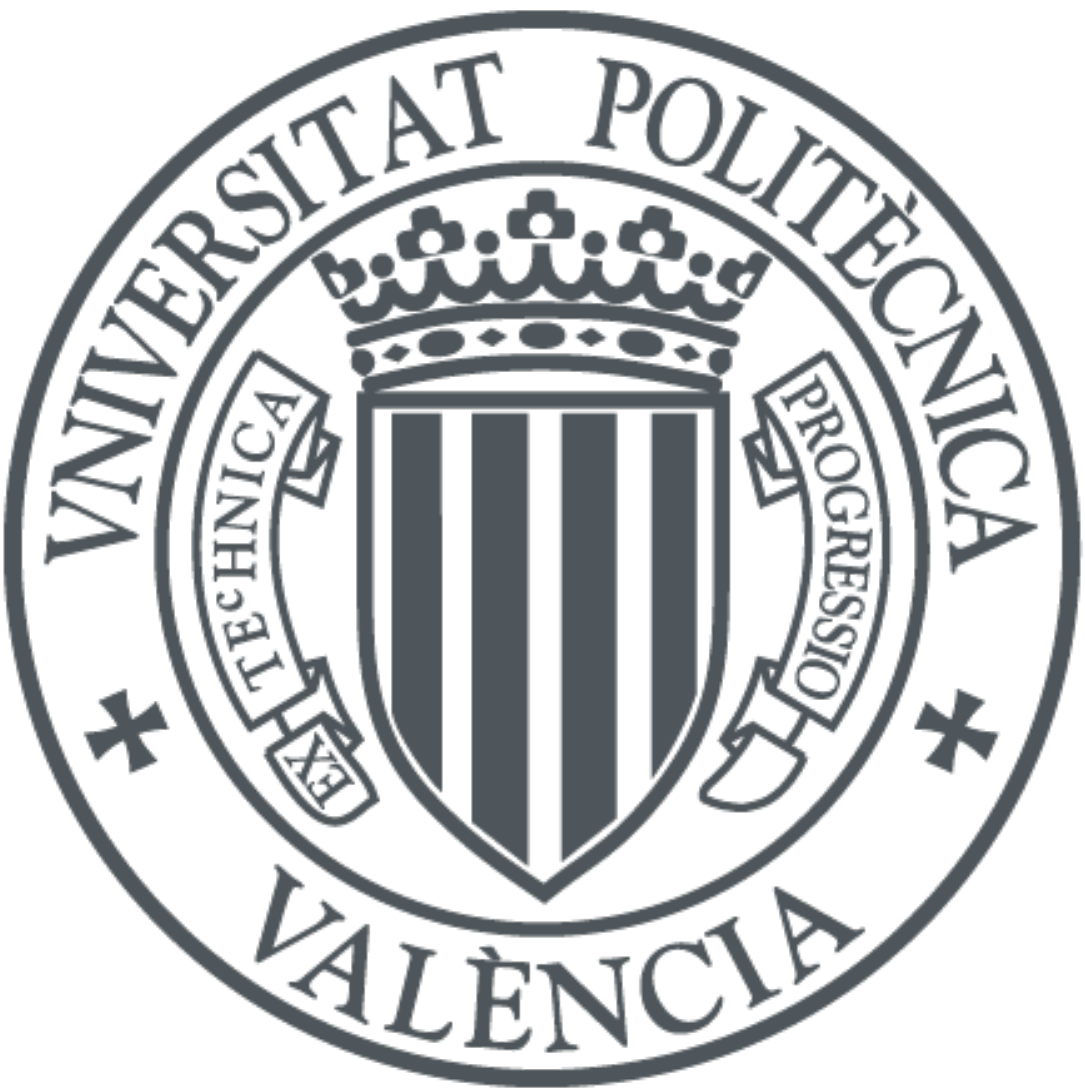

The final publication is available at

https://doi.org/10.1080/03081079.2018.1552687

Copyright Taylor \& Francis

Additional Information

"This is an Accepted Manuscript of an article published by Taylor \& Francis in International Journal of General Systems on 01 Dec 2018, available online:

https://doi.org/10.1080/03081079.2018.1552687" 


\title{
ARTICLE
}

\section{Fuzzy partial metric spaces}

\author{
Valentín Gregori $^{\mathrm{a}}$, Juan-José Miñana ${ }^{\mathrm{b}}$ and David Miravet ${ }^{\mathrm{a}}$ \\ anstituto de Investigación para la Gestión Integrada de Zonas Costeras, Universitat \\ Politècnica de València, Campus de Gandia, Calle Paranimf 1, 46730 Gandia (SPAIN) \\ b Departament de Ciències Matemàtiques i Informàtica, Universitat de les Illes Balears, \\ Carretera de Valldemossa km. 7.5, 07122 Palma (SPAIN)
}

\begin{abstract}
ARTICLE HISTORY
Compiled November 16, 2018

ABSTRACT

In this paper we provide a concept of fuzzy partial metric space $(X, P, *)$ as an extension to fuzzy setting in the sense of Kramosil and Michalek, of the concept of partial metric due to Matthews. This extension has been defined using the residuum operator $\rightarrow_{*}$ associated to a continuous $t$-norm $*$ and without any extra condition on $*$. Similarly, it is defined the stronger concept of $G V$-fuzzy partial metric (fuzzy partial metric in the sense of George and Veeramani). After defining a concept of open ball in $(X, P, *)$, a topology $\mathcal{T}_{P}$ on $X$ deduced from $P$ is constructed, and it is showed that $\left(X, \mathcal{T}_{P}\right)$ is a $T_{0}$-space.
\end{abstract}

\section{KEYWORDS}

Partial metric space; fuzzy metric space; triangular norm; residuum operator.

\section{Introduction}

One can find several generalizations of the concept of metric space in the literature. For instance, in Matthews (1994) it was introduced the notion of partial metric, a generalized metric for which the self-distance is not necessarily zero. It is worth mentioning that, a partial metric $p$ on a non-empty set $X$ induces a topology $\mathcal{T}(p)$ on $X$ which is $T_{0}$. Interesting notes on it can be seen in Bukatin et al. (2014). Another generalization of classical metric, from a different point of view, was given in Menger (1942). In it, Menger gave the concept of statistical metric space, which was developed in Schweizer and Sklar (1960), and renamed as $P M$-space (probabilistic metric space). This concept of $P M$-space was extended to the fuzzy setting in Kramosil and Michalek (1975), who introduced the concept of fuzzy metric. Nowadays, we refer to a fuzzy metric using a more general concept than the one provided in Kramosil and Michalek (1975), that was given in Grabiec (1988) (see also George and Veeramani (1994)), using continuous $t$-norm. Later on, in George and Veeramani (1994) it was introduced a stronger concept than fuzzy metric which we call $G V$-fuzzy metric (Definition 2.7). A ( $G V$-)fuzzy metric $M$ on a set $X$ induces a topology $\mathcal{T}_{M}$ on $X$, and this topology is metrizable (see George and Veeramani (1995); Gregori and Romaguera (2000)). Since both concepts of fuzzy metrics were defined, several authors have contributed to their study from the mathematical point of view (see, for instance, the

CONTACT Juan-José Miñana. Email: jj.minana@uib.es 
following current references Gutiérrez-Gracía et al. (2018); Miñana and Valero (2018); Shukla et al. (2016), and references therein). Moreover, fuzzy metrics have been used successfully in engineering applications such as colour image filtering (see Camarena et al. (2010), and references therein) and perceptual colour difference (see Grečova and Morillas (2016); Gregori et al. (2012)).

In the last years, different works have tried to unify both aforesaid generalizations of classical metric, partial metric and fuzzy one, in a single one notion. For instance, in Yue and $\mathrm{Gu}$ (2014) was given a concept of fuzzy partial metric, which was defined using the continuous minimum $t$-norm. Furthermore, the authors endowed a fuzzifying topology (see Ying (1991)) to the set deduced from the fuzzy partial metric. Later on, in Yue (2015) it was introduced the concept of probabilistic partial metric as a generalization of both fuzzy metrics and partial ones by means of $\Delta^{+}$-valued sets. A probabilistic partial metric is a generalization of the fuzzy partial metric given in Yue and $\mathrm{Gu}$ (2014). Besides, Y. Yue defined the concept of open ball in a probabilistic partial metric space $(X, P, \wedge)$, where $\wedge$ is the minimum $t$-norm, and proved that the family of open balls induces a (classical) topology $\mathcal{T}_{P}$ on $X$. He also proved fixed point theorems on a complete probabilistic partial metric space $(X, P, \wedge)$, and generalized fixed point theorems given in Grabiec (1988) and in Gregori and Sapena (2002). Recently, in Yue and $\mathrm{Gu}(2017)$ the poset $B X$ of formal balls has been studied in these last one spaces, renamed as fuzzy partial metric spaces. Moreover, in Sedghi et al. (2015) has been introduced a concept of partial fuzzy metric which generalizes the concept of strong (non-Archimedean) $G V$-fuzzy metric (see Gregori et al. (2010); Mihet (2008)), and it was proved fixed point theorems for complete partial fuzzy metric spaces.

In this paper we approach the concept of $(G V$-)fuzzy partial metric as an extension of the concept of partial metric to the fuzzy setting in the sense of Kramosil and Michalek and in the George and Veeramani's one. These extensions have been made in a natural way, but for establishing the triangle inequality we have used the residuum operator $\rightarrow_{*}$ associated to a continuous $t$-norm (Definition 2.1). This way of proceeding has been inspired by Demirci (Demicri 2012, Definition 13), without assuming any additional restrictions on the continuous $t$-norm. As in the case of fuzzy metrics a $G V$-fuzzy partial metric can be regarded as a fuzzy partial metric (in the sense of Kramosil and Michalek). Then, in Definition 13 we have defined the concept of open ball $B_{P}(x, r, t)$ centered at $x \in X$, with radius $\left.r \in\right] 0,1[$ and $t>0$, and we have proved that the family $\mathcal{B}=\left\{B_{P}(x, r, t): x \in X, r \in\right] 0,1[, t \in] 0, \infty[\}$ is a base for a topology $\mathcal{T}_{P}$ on $X$ and $\left(X, \mathcal{T}_{P}\right)$ is a $T_{0}$-space (Theorem 4.6). The possibility of refining the concept of open ball in order to obtain a topology on $X$ has been posed as a question (Question 4.7), at the end of Section 4, followed by a justifying remark. The theory is supported with several examples throughout the paper.

The structure of the paper is as follows. In Section 2 we give some preliminary results referred to the residuum operator associated to a continuous $t$-norm and also about fuzzy metrics and partial ones. In Section 3 we introduce and study the concept of fuzzy partial metric. Finally, in Section 4 we deduce, from a fuzzy partial metric $P$ on $X$, a topology $\mathcal{T}_{P}$ on $X$.

\section{Preliminaries}

We have divided this section in two different subsections. In the first one, we recall the basic necessaries on the $t$-norms and the residuum operator related to a $t$-norm. In 
the second one, we include the concepts of fuzzy metric and partial metric and some comments on them.

\subsection{Lattice-ordered monoids and continuous t-norms}

In this subsection, we will recall some notions and results related to lattice-ordered monoids. They will be useful in order to introduce a binary operator that we will use in our definition of fuzzy partial metric space. We begin recalling some concepts given in Birkhoff (1973); Höhle and Klement (1995). They can be found in Klement et al. (2000).

Definition 2.1. Let $(L, \preceq)$ be a lattice and $(L, *)$ a semigroup with neutral element.

(i) The triple $(L, *, \preceq)$ is called a lattice-ordered monoid (or an $l$-monoid) if for all $x, y, z \in L$ we have

(LM1) $x *(y \vee z)=(x * y) \vee(x * z)$,

(LM1) $(x \vee y) * z=(x * z) \vee(y * z)$.

(ii) An $l$-monoid $(L, *, \preceq)$ is said to be commutative if the semigroup $(L, *)$ is commutative.

(iii) A commutative $l$-monoid $(L, *, \preceq)$ is called a commutative, residuated l-monoid if there exists a further binary operation $\rightarrow_{*}$ on $L$ such that for each $x, y, z \in L$ we have

$$
x * y \preceq z \text { if and only if } x \preceq y \rightarrow_{*} z \text {. }
$$

In this case, $\rightarrow_{*}$ is called the $*$-residuum. Moreover, in Klement et al. (2000) it was observed that in each commutative, residuated $l$-monoid $(L, *, \preceq)$, the $*$-residuum operator $\rightarrow_{*}$ is uniquely determined by the formula

$$
x \rightarrow_{*} y=\sup \{z \in L: x * z \preceq y\}
$$

(iv) An $l$-monoid $(L, *, \preceq)$ is called integral if there is a greatest element in the lattice $(L, \preceq)$ which coincides with the neutral element of the semigroup $(L, *)$.

(v) A commutative integral $l$-monoid $(L, * \preceq)$ is called divisible if for each $x, y \in L$ with $y \preceq x$ there exists $z \in L$ such that $x * z=y$.

The concepts introduced in Definition 2.1 are very related with the notion of triangular norm (briefly, $t$-norm). Recall that a $t$-norm is a binary operation $*$ on the unit interval $[0,1]$ such that it is commutative, associative, monotone, and satisfies that $x * 1=x$ for every $x \in[0,1]$.

The most commonly used continuous $t$-norms in Fuzzy Logic are the minimum, denoted by $\wedge$, the usual product, denoted by $*_{P}$, and the Lukasievicz $t$-norm, denoted by $* \mathfrak{L}$, where $x * \mathfrak{L} y=\max \{0, x+y-1\}$.

The aforementioned relation was established in the following proposition provided in Klement et al. (2000).

Proposition 2.2. For each binary operation $*:[0,1] \times[0,1] \rightarrow[0,1]$ the following are equivalent:

(i) $([0,1], *, \leq)$ is a commutative residuated integral $l$-monoid.

(ii) $*$ is a left-continuous $t$-norm. 
Moreover, in Klement et al. (2000) it was pointed out the next corollary of the last result.

Corollary 2.3. $*$ is a continuous $t$-norm if and only if $([0,1], *, \leq)$ is a commutative residuated divisible integral l-monoid.

Taking into account the formula (1), an immediate consequence of the last corollary is that the $*$-residuum operator $\rightarrow_{*}$ of a continuous $t$-norm $*$ is uniquely determined now by the formula

$$
x \rightarrow_{*} y= \begin{cases}1, & \text { if } x \leq y \\ \sup \{z \in L: x * z=y\}, & \text { if } x>y\end{cases}
$$

Attending to this last expression, the *-residuum operator of the minimum, the usual product and the Lukasievicz $t$-norms, respectively, are the following:

$$
\begin{gathered}
x \rightarrow \wedge y= \begin{cases}1, & \text { if } x \leq y ; \\
y, & \text { if } x>y .\end{cases} \\
x \rightarrow_{*_{P}} y= \begin{cases}1, & \text { if } x \leq y ; \\
\frac{y}{x}, & \text { if } x>y .\end{cases} \\
x \rightarrow_{*_{\mathfrak{L}}} y= \begin{cases}1, & \text { if } x \leq y ; \\
1+y-x, & \text { if } x>y .\end{cases}
\end{gathered}
$$

In Klement et al. (2000) it was also established a representation theorem of a (large) class of $t$-norms, which are the Archimedean and continuous ones. Recall that a $t$-norm is called Archimedean if for each $x, y \in[0,1]$ there exists $n \in \mathbb{N}$ with $x * \cdots * *^{(n)} x<y$, where $* \cdots * *^{(n)}$ denotes the $n$-times composition by $*$. Two well-known examples of Archimedean $t$-norms are the usual product and the Lukasievicz one. Nevertheless, the minimum $t$-norm is an example of non-Archimedean one.

In order to obtain the aforesaid representation, it was introduced the following concept.

Definition 2.4. An additive generator $f_{*}:[0,1] \rightarrow[0, \infty]$ of a $t$-norm $*$ is a strictly decreasing function which is right-continuous at 0 , satisfying $f_{*}(1)=0$, and such that for $x, y \in[0,1]$ we have

$$
f_{*}(x)+f_{*}(y) \in \operatorname{Ran}\left(f_{*}\right) \cup\left[f_{*}(0), \infty\right]
$$

and also

$$
x * y=f_{*}^{(-1)}\left(f_{*}(x)+f_{*}(y)\right), \text { for all } x, y \in[0,1],
$$

where $f_{*}^{(-1)}$ denotes the pseudo-inverse of the function $f_{*}$ (see Klement et al. (2000)).

Now, we present the announced representation theorem. 
Theorem 2.5. A binary operator $*$ in $[0,1]$ is a continuous Archimedean $t$-norm if and only if there exists a continuous additive generator $f_{*}$ of $*$.

Moreover, an additive generator $f_{*}$ of a continuous Archimedean $t$-norm $*$ allows us to obtain a simpler formula of the $*$-residuum, as follows:

$$
x \rightarrow_{*} y= \begin{cases}1, & \text { if } x=0 \\ f_{*}^{(-1)}\left(\max \left\{f_{*}(y)-f_{*}(x), 0\right\}\right), & \text { if } x, y \in] 0,1] .\end{cases}
$$

Note that the pseudo-inverse of a continuous additive generator $f_{*}$ is given by

$$
f_{*}^{(-1)}(y)=f_{*}^{-1}\left(\min \left\{f_{*}(0), y\right\}\right) .
$$

Remark 1. Attending to Equation (7), it is obvious that given a continuous Archimedean $t$-norm $*$, its $*$-residuum $\rightarrow_{*}$ is continuous on $\left.\left.\left.] 0,1\right] \times\right] 0,1\right]$. Nevertheless, one can easily observe that the last affirmation is not true, in general, when we consider continuous non-Archimedean $t$-norms, as it is so the minimum $t$-norm (see expression (3)).

To finish this subsection, we recall another well-known continuous Archimedean $t$ norm called the Hamacher product. It will be denoted by $*_{H}$ and it is given by the following expression:

$$
a *_{H} b= \begin{cases}0, & \text { if } a=b=0 \\ \frac{a b}{a+b-a b}, & \text { elsewhere }\end{cases}
$$

for each $a, b \in[0,1]$.

In Klement et al. (2000) it was pointed out that the function $f_{H}(x)=\frac{1-x}{x}$ is an additive generator of $*_{H}$ and so, on account of formula (8), the function $f_{H}^{(-1)}(y)=\frac{1}{1+y}$ is its pseudo-inverse. Attending to these observations and taking into account the formula (7), the expression of the $*_{H}$-residuum is given by

$$
x \rightarrow_{*_{H}} y= \begin{cases}1, & \text { if } x \leq y \\ \frac{x y}{x y+x-y}, & \text { if } x>y .\end{cases}
$$

\section{2. $\quad$ Fuzzy metric and partial metric spaces}

In this last subsection, we compile the basics on fuzzy metrics and partial metrics.

Definition 2.6 (Grabiec (1988); Kramosil and Michalek (1975)). A fuzzy metric space, in the sense of Kramosil and Michalek is an ordered triple $(X, M, *)$ such that $X$ is a (non-empty) set, $*$ is a continuous $t$-norm and $M$ is a fuzzy set on $X \times X \times[0, \infty[$, satisfying the following conditions, for all $x, y, z \in X$ and $s, t \in] 0, \infty[$ :

(KM1) $M(x, y, 0)=0$;

(KM2) $M(x, y, t)=1$ for all $t>0$ if and only if $x=y$;

(KM3) $M(x, y, t)=M(y, x, t)$;

(KM4) $M(x, y, t) * M(y, z, s) \leq M(x, z, t+s)$; 
(KM5) The function $\left.M_{x, y}:\right] 0, \infty\left[\rightarrow[0,1]\right.$ is left-continuous, where $M_{x, y}(t)=$ $M(x, y, t)$ for each $t \in] 0, \infty[$.

Later, in George and Veeramani (1994) it was modified the above concept as follows.

Definition 2.7 (George and Veeramani (1994)). A $G V$-fuzzy metric space is an ordered triple $(X, M, *)$ such that $X$ is a (non-empty) set, $*$ is a continuous $t$-norm and $M$ is a fuzzy set on $X \times X \times] 0, \infty$ [ satisfying, for all $x, y, z \in X$ and $s, t \in] 0, \infty[$, conditions (KM3), (KM4) and the following ones:

(GV1) $M(x, y, t)>0$;

(GV2) $M(x, y, t)=1$ if and only if $x=y$;

(GV5) The function $\left.\left.M_{x, y}:\right] 0, \infty[\rightarrow] 0,1\right]$ is continuous.

If $(X, M, *)$ is a $(\mathrm{GV}$-)fuzzy metric space, we say that $(M, *)$, or simply $M$, is a (GV-)fuzzy metric on $X$. A $G V$-fuzzy metric $M$ can be regarded as a fuzzy metric defining $M(x, y, 0)=0$ for each $x, y \in X$.

Remark 2. In the notion of fuzzy metric the $t$-norm just takes part in (KM4), and so, attending to this axiom, if $(M, *)$ is a fuzzy metric on $X$, then $(M, \diamond)$ is so whenever $\diamond$ is a continuous $t$-norm smaller that $*$, i.e. if for each $a, b \in[0,1]$ we have that $a * b \geq a \diamond b$.

Every fuzzy metric $M$ on $X$ generates a metrizable topology $\mathcal{T}_{M}$ on $X$ which has as a base the family of open balls

$$
\mathcal{B}=\left\{B_{M}(x, \epsilon, t): x \in X, \epsilon \in\right] 0,1[, t \in] 0, \infty[\},
$$

where $B_{M}(x, \epsilon, t)=\{y \in X: M(x, y, t)>1-\epsilon\}$ for all $\left.x \in X, \epsilon \in\right] 0,1[$ and $t \in] 0, \infty[$.

The following are two well-known examples of $G V$-fuzzy metric spaces, both defined from a classical metric.

Let $(X, d)$ be a metric space. Define the fuzzy sets $M_{e}$ and $M_{d}$, respectively, on $X \times X \times] 0, \infty[$ as follows

$$
\begin{gathered}
M_{e}(x, y, t)=e^{-\frac{d(x, y)}{t}}, \\
M_{d}(x, y, t)=\frac{t}{t+d(x, y)} .
\end{gathered}
$$

Then, $\left(X, M_{e}, \wedge\right)$ and $\left(X, M_{d}, \wedge\right)$ are $G V$-fuzzy metric spaces, and so both $M_{e}$ and $M_{d}$ are $G V$-fuzzy metrics on $X$ for each continuous $t$-norm, since $\wedge \geq *$ for every continuous $t$-norm $*$. Furthermore, it is well-known that the topologies $\mathcal{T}_{M_{e}}$ and $\mathcal{T}_{M_{d}}$ coincide with the topology $\mathcal{T}(d)$ induced by $d$ on $X$.

The fuzzy metric $M_{d}$ is called the standard fuzzy metric induced by $d$.

Definition 2.8 (Matthews (1994)). A partial metric space is a pair $(X, p)$, where $X$ is a non-empty set and $p$ is a function on $X \times X$ such that for all $x, y, z \in X$ :

(P1) $0 \leq p(x, x) \leq p(x, y)$;

(P2) $p(x, x)=p(x, y)=p(y, y) \Leftrightarrow x=y$;

(P3) $p(x, y)=p(y, x)$; 
Remark 3. Notice that (P4) can be written as

$$
p(x, z)-p(x, x) \leq p(x, y)-p(x, x)+p(y, z)-p(y, y) .
$$

Every partial metric $p$ on $X$ generates a topology $\mathcal{T}(p)$ on $X$ which has as a base the family of open balls,

$$
\mathcal{B}=\left\{B_{p}(x ; \varepsilon): x \in X, \varepsilon \in\right] 0, \infty[\},
$$

and $(X, \mathcal{T}(p))$ is a $T_{0}$-space.

Recall that the non-empty open ball centred at $x \in X$ and radius $\varepsilon>0$ is defined by

$$
B_{p}(x ; \varepsilon)=\{y \in X: p(x, y)<p(x, x)+\varepsilon\} .
$$

\section{Fuzzy partial metric spaces}

The aim of this section is to extend properly the concept of partial metric to the fuzzy setting. First, we will introduce the concept of $(G V$-)fuzzy partial metric by means of continuous $t$-norms, which will be according to the given concept of (GV-)fuzzy metric.

Definition 3.1. A fuzzy partial metric space is an ordered triple $(X, P, *)$ such that $X$ is a (non-empty) set, $*$ is a continuous $t$-norm and $P$ is a fuzzy set on $X \times X \times[0, \infty[$ satisfying the following conditions, for all $x, y, z \in X$ and $s, t \in] 0, \infty[$ :

(FPKM0) $P(x, y, 0)=0$;

(FPKM1) $P(x, y, t) \leq P(x, x, t)$;

(FPKM2) $P(x, x, t)=P(y, y, t)=P(x, y, t)$, for each $t \in] 0, \infty[$, if and only if $x=y$;

(FPKM3) $P(x, y, t)=P(y, x, t)$;

(FPKM4) $P(x, x, t+s) \rightarrow_{*} P(x, z, t+s) \geq$ $\left(P(x, x, t) \rightarrow_{*} P(x, y, t)\right) *\left(P(y, y, s) \rightarrow_{*} P(y, z, s)\right)$;

(FPKM5) The assignment $\left.P_{x, y}:\right] 0, \infty\left[\rightarrow[0,1]\right.$, given by $P_{x, y}(t)=P(x, y, t)$, is a left-continuous function.

According to the notion of $G V$-fuzzy metric space we introduce the following definition.

Definition 3.2. A $G V$-fuzzy partial metric space is an ordered triple $(X, P, *)$ such that $X$ is a (non-empty) set, $*$ is a continuous $t$-norm and $P$ is a fuzzy set on $X \times X \times] 0, \infty$ [ satisfying, for all $x, y, z \in X$ and $s, t \in] 0, \infty$ [, conditions (FPKM3), (FPKM4) and the following ones:

(FPGV1) $0<P(x, y, t) \leq P(x, x, t)$;

(FPGV2) $P(x, x, t)=P(y, y, t)=P(x, y, t)$ if and only if $x=y$;

(FPGV5) The assignment $\left.\left.P_{x, y}:\right] 0, \infty[\rightarrow] 0,1\right]$, given by $P_{x, y}(t)=P(x, y, t)$, is a continuous function.

If $(X, P, *)$ is a $(G V$-)fuzzy partial metric space we will say that $(P, *)$, or simply 
$P$, is a $(G V$-) fuzzy partial metric on $X$.

Remark 4. As in the case of fuzzy metrics, a $G V$-fuzzy partial metric can be regarded as a fuzzy partial one defining $P(x, y, 0)=0$ for each $x, y \in X$. The purpose of this paper is to introduce a topology on a non-empty set $X$ deduced from a $(G V$-)fuzzy partial metric on $X$. Now, as the reader will be able to observe, the value $P(x, y, 0)$ does not play any role in our construction and then the results that we will state for fuzzy partial metric spaces will be also valid for $G V$-fuzzy partial metrics.

Note that the above notions are generalizations of the concepts of fuzzy metric space and $G V$-fuzzy metric space, respectively. Indeed, one can show that a fuzzy metric $(M, *)$ on a non-empty set $X$ is a fuzzy partial metric satisfying the additional condition below, for each $x \in X$ :

$$
M(x, x, t)=1 \text { for each } t \in] 0, \infty[.
$$

After introducing both approaches, to the fuzzy context, of the notion of partial metric, we will justify the axiomatic that has been chosen for them. We only make such observations on the notion of fuzzy partial metric space, since for the George and Veeramani's approach they can be deduced similarly.

Remark 5. One can observe that the axioms (FPKM1), (FPKM2) and (FPKM3) are "literal" adaptations to the fuzzy context (following the ideas of the notion of fuzzy metric space introduced by Kramosil and Michalek) of axioms (P1), (P2) and (P3), respectively. Attending to Remark 3, the (FPKM4) axiom is an adaptation of the inequality (P4). Finally, we have included axiom (FPKM5) for the sake of similarity with fuzzy metric spaces.

After justifying the axioms chosen in the definition of fuzzy partial metric, we continue our study introducing two propositions that provide two examples of $G V$ fuzzy partial metric spaces, which generalize, in some sense, the $G V$-fuzzy metric spaces recalled in expressions (10) and (11), respectively.

Proposition 3.3. Let $(X, p)$ be a partial metric space. We define the fuzzy set on $X \times X \times] 0, \infty[$ as follows

$$
P_{e}(x, y, t)=e^{-\frac{p(x, y)}{t}} .
$$

Then, $\left(X, P_{e}, *_{P}\right)$ is a $G V$-fuzzy partial metric space.

Proof. Let $(X, p)$ be a partial metric space and let $P_{e}$ the function given by

$$
\left.P_{e}(x, y, t)=e^{-\frac{p(x, y)}{t}}, \text { for each } x, y \in X, t \in\right] 0, \infty[.
$$

Let $x, y, z \in X$ and $t, s \in] 0, \infty\left[\right.$. We will see that $P_{e}$ satisfies all the axioms of Definition 3.2.

(FPGV1) Note that $0<e^{-\frac{p(x, y)}{t}} \leq e^{-\frac{p(x, x)}{t}} \leq 1$, since $0 \leq p(x, x) \leq p(x, y)$.

(FPGV2) On the one hand, $P_{e}(x, x, t)=P_{e}(y, y, t)=P_{e}(x, y, t)$ implies $e^{-\frac{p(x, x)}{t}}=$ $e^{-\frac{p(y, y)}{t}}=e^{-\frac{p(x, y)}{t}}$. Then, $p(x, x)=p(y, y)=p(x, y)$ and so $x=y$. On the 
other hand, if $x=y$, it is obvious that $P_{e}(x, x, t)=P_{e}(y, y, t)=P_{e}(x, y, t)$.

(FPKM3) It is obvious, since $p(x, y)=p(y, x)$.

(FPKM4) Recall that the function $f_{P}$, where $f_{P}(x)=-\log (x)$ for each $x \in[0,1]$, is an additive generator of the usual product $t$-norm (it was observed in Klement et al. (2000)). Now, an easy computation shows that the function $f_{P}^{(-1)}$, given by $f_{P}^{(-1)}(y)=e^{-y}$ for each $y \in\left[0, \infty\left[\right.\right.$, is the pseudo-inverse of $f_{P}$.

Let $u, v \in X$ and $r \in] 0, \infty[$. Attending to formula (7) in Section 2.1 we have that

$$
\begin{aligned}
P_{e}(u, u, r) \rightarrow_{*_{P}} & P_{e}(u, v, r)=f_{P}^{(-1)}\left(f_{P}\left(P_{e}(u, v, r)\right)-f_{P}\left(P_{e}(u, u, r)\right)\right)= \\
= & f_{P}^{(-1)}\left(-\log \left(e^{-\frac{p(u, v)}{r}}\right)+\log \left(e^{-\frac{p(u, u)}{r}}\right)\right)= \\
& f_{P}^{(-1)}\left(\frac{p(u, v)}{r}-\frac{p(u, u)}{r}\right)=e^{-\frac{p(u, v)-p(u, u)}{r}} .
\end{aligned}
$$

Then, using Remark 3 we have that

$$
\begin{gathered}
P_{e}(x, x, t+s) \rightarrow_{*_{P}} P_{e}(x, z, t+s)=e^{-\frac{p(x, z)-p(x, x)}{t+s}} \geq e^{-\frac{p(x, y)-p(x, x)+p(y, z)-p(y, y)}{t+s}} \geq \\
\geq e^{-\frac{p(x, y)-p(x, x)+p(y, z)-p(y, y)}{\max \{t, s\}}} \geq e^{-\frac{p(x, y)-p(x, x)}{t}} *_{P} e^{-\frac{p(y, z)-p(y, y)}{s}}= \\
=\left(P_{e}(x, x, t) \rightarrow_{*_{P}} P_{e}(x, y, t)\right) *_{P}\left(P_{e}(y, y, s) \rightarrow_{*_{P}} P_{e}(y, z, s)\right) .
\end{gathered}
$$

(FPGV5) Obviously, the function $\left(P_{e}\right)_{x, y}(t)=e^{-\frac{p(x, y)}{t}}$ is a continuous function on $] 0, \infty[$, for each $x, y \in X$.

Hence, $\left(X, P, *_{P}\right)$ is a $G V$-fuzzy partial metric space.

Following the same ideas of the last proof one can prove the following proposition.

Proposition 3.4. Let $(X, p)$ be a partial metric space. We define the fuzzy set on $X \times X \times] 0, \infty[$

$$
P_{d}(x, y, t)=\frac{t}{t+p(x, y)}
$$

Then, $\left(X, P_{d}, *_{H}\right)$ is a $G V$-fuzzy partial metric space.

Moreover, we could show that both examples, presented in Proposition 3.3 and Proposition 3.4, are $G V$-fuzzy partial metrics for the minimum $t$-norm too. Nevertheless, in the next example we will show that the fuzzy set $P_{d}$ introduced in Proposition 3.4 is not a fuzzy partial metric on $X$ for the Lukasievicz $t$-norm, in general.

Example 3.5. Let $(X, p)$ be the partial metric space, where $X=[0, \infty[$ and $p(x, y)=\max \{x, y\}$ for each $x, y \in X$, and consider the fuzzy set $P_{d}$ on $\left.X \times X \times\right] 0, \infty[$ 
given by $P_{d}(x, y, t)=\frac{t}{t+p(x, y)}$. We will see that $\left(X, P_{d}, *_{\mathfrak{L}}\right)$ is not a $G V$-fuzzy partial metric space. Indeed, we will show that $P_{d}$ does not satisfy axiom (FPKM4) for the Lukasievicz $t$-norm.

Let $u, v \in X$ and $r \in] 0, \infty[$. By formula (5), we have that

$$
P_{d}(u, u, r) \rightarrow_{*_{\mathfrak{L}}} P_{d}(u, v, r)=1+\frac{r}{r+\max \{u, v\}}-\frac{r}{r+u} .
$$

Now, consider $x=1 ; y=2 ; z=10$ and $t=s=1$. By the last expression,

$$
P_{d}(x, x, t+s) \rightarrow_{*_{\mathfrak{L}}} P_{d}(x, z, t+s)=1+\frac{1+1}{1+1+10}-\frac{1+1}{1+1+1}=1+\frac{1}{6}-\frac{2}{3}=\frac{1}{2},
$$

and

$$
\begin{gathered}
\left(P_{d}(x, x, t) \rightarrow_{*_{\mathfrak{L}}} P_{d}(x, y, t)\right) *_{\mathfrak{L}}\left(P_{d}(y, y, s) \rightarrow_{*_{\mathfrak{L}}} P_{d}(y, z, s)\right)= \\
=\left(1+\frac{1}{1+2}-\frac{1}{1+1}\right)+\left(1+\frac{1}{1+10}-\frac{1}{1+2}\right)-1=\frac{1}{2}+\frac{1}{11} .
\end{gathered}
$$

Therefore, in this case, we have that $P_{d}(x, x, t+s) \rightarrow_{*_{\mathfrak{L}}} P_{d}(x, z, t+s)<$ $\left(P_{d}(x, x, t) \rightarrow_{*_{\mathfrak{L}}} P_{d}(x, y, t)\right) *_{\mathfrak{L}}\left(P_{d}(y, y, s) \rightarrow_{*_{\mathfrak{L}}} P_{d}(y, z, s)\right)$.

Remark 6. Since $*_{H} \geq *_{\mathcal{L}}$, the preceding example shows a significant difference between fuzzy metrics and fuzzy partial ones. Indeed, given a fuzzy partial metric space $(X, P, *)$ it is not true, in general, that the tern $(X, P, \diamond)$ has to be also a fuzzy partial metric space, whenever $\diamond \leq *$.

The following example shows a fuzzy partial metric which is not explicitly deduced from a classical partial metric.

Example 3.6. Let $X=] 0,1]$. We define the fuzzy set $P$ on $X \times X \times] 0, \infty[$ by the next expression

$$
P(x, y, t)= \begin{cases}\min \{x, y\} \cdot \frac{t^{2}}{t+1}, & \text { if } x \neq y, t \in] 0,1] \\ \min \{x, y\} \cdot \frac{t}{t+1}, & \text { elsewhere }\end{cases}
$$

We will see that $(X, P, \wedge)$ is a fuzzy partial metric space.

(FPKM0) By definition, $P(x, y, 0)=\min \{x, y\} \cdot \frac{0}{1}=0$ for every $x, y \in X$.

(FPKM1) Take $x, y \in X$ with $x \neq y$. We will distinguish two cases.

(1) Suppose that $t \in] 0,1]$ 0 .

First, note that $P(x, x, t)=x \cdot \frac{t}{t+1}<1$ and $P(x, y, t)=\min \{x, y\} \cdot \frac{t^{2}}{t+1}>$ Then,

$$
1>P(x, x, t)=x \cdot \frac{t}{t+1} \geq \min \{x, y\} \cdot \frac{t^{2}}{t+1}=P(x, y, t)>0 .
$$

(2) Now, suppose that $t \in] 1, \infty\left[\right.$. Then, $P(x, x, t)=x \cdot \frac{t}{t+1}<1$ and $P(x, y, t)=$ 
$\min \{x, y\} \cdot \frac{t}{t+1}>0$, and so

$$
1>P(x, x, t)=x \cdot \frac{t}{t+1} \geq \min \{x, y\} \cdot \frac{t}{t+1}=P(x, y, t)>0 .
$$

Thus, for each $x, y \in X$ and $t \in] 0, \infty[$ it is satisfied $0<P(x, y, t) \leq P(x, x, t) \leq 1$.

(FPKM2) Obviously, if $x=y$, then $P(x, x, t)=P(y, y, t)=P(x, y, t)$. Conversely, let $x, y \in X$ and $t \in] 0, \infty[$ satisfying $P(x, x, t)=P(y, y, t)=P(x, y, t)$. Then,

$$
P(x, x, t)=x \cdot \frac{t}{t+1}=y \cdot \frac{t}{t+1}=P(y, y, t),
$$

and so $x=y$.

(FPKM3) It is obvious by definition of $P$.

(FPKM4) Let $x, y, z \in X$ and $t, s \in] 0, \infty[$. Suppose that $x \neq z$, since if $x=z$ this axiom is clearly held. We will distinguish two possibilities:

(1) Suppose that $t+s \in] 0,1]$. On the one hand, an easy computation shows that

$$
\frac{(t+s)^{2}}{1+t+s} \geq \max \left\{\frac{t^{2}}{1+t}, \frac{s^{2}}{1+s}\right\}
$$

On the other hand,

$$
P(x, x, t+s) \rightarrow \wedge P(x, z, t+s)=\min \{x, z\} \cdot \frac{(t+s)^{2}}{t+s+1} .
$$

Now, if $x=y$ (or similarly, $y=z$ ), then

$$
\left(P(x, x, t) \rightarrow_{\wedge} P(x, y, t)\right) \wedge\left(P(y, y, s) \rightarrow_{\wedge} P(y, z, s)\right)=\min \{x, z\} \cdot \frac{s^{2}}{1+s} .
$$

Thus, (FPKM4) is held.

Contrary, if $x \neq y$ and $y \neq z$, then

$$
\begin{gathered}
(P(x, x, t) \rightarrow \wedge P(x, y, t)) \wedge(P(y, y, s) \rightarrow \wedge P(y, z, s))= \\
\left(\min \{x, y\} \cdot \frac{t^{2}}{1+t}\right) \wedge\left(\min \{y, z\} \cdot \frac{s^{2}}{s+1}\right) \leq \\
\leq\left(\min \{x, y\} \cdot \max \left\{\frac{t^{2}}{1+t}, \frac{s^{2}}{1+s}\right\}\right) \wedge\left(\min \{y, z\} \cdot \max \left\{\frac{t^{2}}{1+t}, \frac{s^{2}}{1+s}\right\}\right) \leq \\
\leq \min \{x, z\} \cdot \max \left\{\frac{t^{2}}{1+t}, \frac{s^{2}}{1+s}\right\} \leq \min \{x, z\} \cdot \frac{(t+s)^{2}}{1+t+s}=
\end{gathered}
$$




$$
=P(x, x, t+s) \rightarrow_{\wedge} P(x, z, t+s),
$$

and so, (FPKM4) is fulfilled too.

(2) Suppose that $t+s \in] 1, \infty[$. In such a case, observe that $x \leq z$ implies $P(x, x, t+s)=P(x, z, t+s)$. Then $P(x, x, t+s) \rightarrow \wedge P(x, z, t+s)=1$ and so, the triangle inequality is held. Thus, suppose that $x>z$. Besides, assume that $y \neq x$ and $y \neq z$, since contrary the inequality is fulfilled obviously. On the one hand,

$$
P(x, x, t+s) \rightarrow \wedge P(x, z, t+s)=z \cdot \frac{t+s}{t+s+1} .
$$

On the other hand, we claim that $\left(P(x, x, t) \rightarrow_{\wedge} P(x, y, t)\right) \wedge$ $(P(y, y, s) \rightarrow \wedge P(y, z, s)) \leq \min \{y, z\} \cdot \max \left\{\frac{t}{t+1}, \frac{s}{s+1}\right\}$. To show it, we will distinguish three cases:

(a) Suppose that $y>x$. Then,

$$
P(x, x, t) \rightarrow \wedge P(x, y, t)= \begin{cases}x \cdot \frac{t^{2}}{t+1}, & \text { if } x \neq y, t \in] 0,1] \\ 1, & \text { elsewhere }\end{cases}
$$

and

$$
P(y, y, s) \rightarrow \wedge P(y, z, s)= \begin{cases}z \cdot \frac{s^{2}}{s+1}, & \text { if } x \neq y, s \in] 0,1] \\ z \cdot \frac{s}{s+1}, & \text { elsewhere }\end{cases}
$$

Then, $\left(P(x, x, t) \rightarrow_{\wedge} P(x, y, t)\right) \wedge\left(P(y, y, s) \rightarrow_{\wedge} P(y, z, s)\right) \leq z \cdot \frac{s}{s+1} \leq$ $\min \{y, z\} \cdot \max \left\{\frac{t}{t+1}, \frac{s}{s+1}\right\}$.

(b) Suppose that $x>y>z$. Then,

$$
P(x, x, t) \rightarrow \wedge P(x, y, t)= \begin{cases}y \cdot \frac{t^{2}}{t+1}, & \text { if } x \neq y, t \in] 0,1] \\ y \cdot \frac{t}{t+1}, & \text { elsewhere }\end{cases}
$$

and

$$
P(y, y, s) \rightarrow_{\wedge} P(y, z, s)= \begin{cases}z \cdot \frac{s^{2}}{s+1}, & \text { if } x \neq y, s \in] 0,1] \\ z \cdot \frac{s}{s+1}, & \text { elsewhere }\end{cases}
$$

Then, $\left(P(x, x, t) \rightarrow_{\wedge} P(x, y, t)\right) \wedge\left(P(y, y, s) \rightarrow_{\wedge} P(y, z, s)\right) \leq z \cdot \frac{s}{s+1} \leq$ $\min \{y, z\} \cdot \max \left\{\frac{t}{t+1}, \frac{s}{s+1}\right\}$.

(c) Suppose that $x>z>y$. Then,

$$
P(x, x, t) \rightarrow_{\wedge} P(x, y, t)= \begin{cases}y \cdot \frac{t^{2}}{t+1}, & \text { if } x \neq y, t \in] 0,1] \\ y \cdot \frac{t}{t+1}, & \text { elsewhere }\end{cases}
$$

and

$$
P(y, y, s) \rightarrow_{\wedge} P(y, z, s)= \begin{cases}y \cdot \frac{s^{2}}{s+1}, & \text { if } x \neq y, s \in] 0,1] \\ 1, & \text { elsewhere }\end{cases}
$$


Then, $\left(P(x, x, t) \rightarrow_{\wedge} P(x, y, t)\right) \wedge\left(P(y, y, s) \rightarrow_{\wedge} P(y, z, s)\right) \leq y$. $\max \left\{\frac{t}{t+1}, \frac{s}{s+1}\right\} \leq \min \{y, z\} \cdot \max \left\{\frac{t}{t+1}, \frac{s}{s+1}\right\}$.

Therefore, since $z \cdot \frac{t+s}{t+s+1} \geq \min \{y, z\} \cdot \max \left\{\frac{t}{t+1}, \frac{s}{s+1}\right\}$ we have that (FPKM4) is satisfied.

(FPKM5) By definition of $P$, it is easy to verify that $P_{x, y}$ given by $P_{x, y}(t)=P(x, y, t)$, is, obviously, a (non-decreasing) continuous function on $[0,+\infty[$, for each $x, y \in X$.

Hence, $(X, P, \wedge)$ is a fuzzy partial metric space.

The next example provides a fuzzy partial metric which is not a $G V$-fuzzy partial one.

Example 3.7. Let $(X, d)$ be a metric space and let $c \in] 0,1]$. We define the fuzzy set on $X \times X \times[0, \infty[$ as follows

$$
P_{0}(x, y, t)= \begin{cases}0, & \text { if } d(x, y) \geq t \\ c, & \text { if } d(x, y)<t\end{cases}
$$

We will see that $\left(X, P_{0}, \wedge\right)$ is a fuzzy partial metric space.

Proof. Let $x, y, z \in X$ and $t, s \in] 0, \infty\left[\right.$. We will prove that $\left(X, P_{0}, \wedge\right)$ satisfies the axioms of Definition 3.1.

$\left(\right.$ FPKM0) $P_{0}(x, y, 0)=0$ since $d(x, y) \geq 0$ for every $x, y \in X$.

(FPKM1) Observe that $P_{0}(x, x, t)=c$, for each $\left.t \in\right] 0, \infty[$, since $d(x, x)=0<t$. Then, for each $x, y \in X$ and $t \in] 0, \infty\left[\right.$ we have that $P_{0}(x, y, t) \leq P_{0}(x, x, t)$.

(FPKM2) Let $x, y \in X$. On the one hand, by the last observation $P_{0}(x, x, t)=P_{0}(y, y, t)=$ $P_{0}(x, y, t)$ for each $\left.t \in\right] 0, \infty\left[\right.$, implies $P_{0}(x, y, t)=c$ for each $\left.t \in\right] 0, \infty[$, and so, by definition of $P_{0}$ we have that $d(x, y)=0$. Thus $x=y$. On the other hand, if $x=y$, it is obvious that $P_{0}(x, x, t)=P_{0}(y, y, t)=P_{0}(x, y, t)$.

(FPKM3) It is obvious, since $d(x, y)=d(y, x)$.

(FPKM4) First of all, attending to formula (3) in Subsection 2.1, by definition of $P_{0}$ we have that

$$
P_{0}(u, u, r) \rightarrow_{\wedge} P_{0}(u, v, r)= \begin{cases}0, & \text { if } d(u, v) \geq r \\ 1, & \text { if } d(u, v)<r\end{cases}
$$

for each $u, v \in X$ and each $r \in] 0, \infty[$.

Let $M_{0}(x, y, t)=P_{0}(x, x, t) \rightarrow \wedge P_{0}(x, y, t)$. Then, given $x, y, z \in X$ and $t, s \in$ ] $0, \infty[$, we have that

$$
M_{0}(x, z, t+s)= \begin{cases}0, & \text { if } d(x, z) \geq t+s \\ 1, & \text { if } d(x, z)<t+s\end{cases}
$$

Therefore, if $d(x, z)<t+s$, the inequality

$$
M_{0}(x, z, t+s) \geq M_{0}(x, y, t) \wedge M(y, z, s)
$$

is fulfilled for every $x, y, z \in X$ and $t, s>0$. Alternatively, if $d(x, y) \geq t+s$, since $d(x, z) \leq d(x, y)+d(y, z)$ for every $x, y, z \in X$, we have that $d(x, y) \geq t$ or 
$d(y, z) \geq s$. In that case, $M_{0}(x, y, t)=0$ or $M_{0}(y, z, s)=0$. Hence, (FPKM4) is also satisfied.

(FPKM5) By definition, the assignment $\left(P_{0}\right)_{x, y}(t)$ is a left-continuous function on $] 0, \infty[$, for each $x, y \in X$.

However, $\left(P_{0}\right)_{x, y}$ is not continuous whenever $x \neq y$, and consequently $P_{0}$ is not a $G V$-fuzzy partial metric.

We finish this section providing another significant difference between $(G V$-)fuzzy metrics and $(G V$-)fuzzy partial metrics. Such a difference is given by the fact that, for each $x, y \in X$, the function $P_{x, y}$ defined in the axiom (FPKM5) is not increasing, in general.

Example 3.8. Consider the set $X=\mathbb{R}$ and the usual product $t$-norm $*_{P}$. We define a fuzzy set $P$ on $X \times X \times[0, \infty[$ given by

$$
P(x, y, t)= \begin{cases}0, & \text { if } t=0 \\ e^{-t}, & \text { if } x=y \\ \frac{1}{2} e^{-t}, & \text { if } x \neq y\end{cases}
$$

It is not hard to check that $\left(X, P, *_{P}\right)$ is a fuzzy partial metric space. Indeed, (FPKM0)(FPKM3) are obviously fulfilled by $P$ attending to its definition. To show (FPKM4), consider $x, y, z \in X$, with $x \neq z$ (since, contrarily, $P(x, x, t+s) \rightarrow_{*_{P}} P(x, z, t+s)=1$ and so, the inequality is satisfied) and $t, s \in] 0, \infty[$. On account of formula (4) in Subsection 2.1,

$$
P(x, x, t+s) \rightarrow_{*_{P}} P(x, z, t+s)=\frac{1}{2} .
$$

Now, by our assumption, at least $x \neq y$ or $z \neq y$, and so

$$
\left(P(x, x, t) \rightarrow_{*_{P}} P(x, y, t)\right) *_{P}\left(P(y, y, s) \rightarrow_{*_{P}} P(y, z, s)\right) \leq \frac{1}{2} .
$$

Therefore, (FPKM4) is also held.

Obviously, $P_{x, y}$ is a continuous function for each $x, y \in X$, which is not increasing.

\section{Topology induced by a fuzzy partial metric}

The aim of this section is to define a topology deduced from a fuzzy partial metric. Such a topology is defined by means of open balls, in a similar way to the classical case. Based on the notions of open ball, both in partial metrics and fuzzy ones, it seems natural to define the concept of open ball in a fuzzy partial metric space as follows:

Definition 4.1. Let $(X, P, *)$ be a fuzzy metric space, the open ball $\tilde{B}_{P}$ centred at $x \in X$, with radius $r \in] 0,1[$ and parameter $t \in] 0, \infty[$ is defined by the next expression:

$$
\tilde{B}_{P}(x, r, t)=\left\{y \in X: P(x, x, t) \rightarrow_{*} P(x, y, t)>1-r\right\} .
$$


Observe that, for each $x, y \in X$, the assignment $\left.P_{x, x, y}:\right] 0, \infty[\rightarrow[0,1]$ is a welldefined function, where $P_{x, x, y}$ is given by

$$
\left.P_{x, x, y}(t)=P(x, x, t) \rightarrow_{*} P(x, y, t), \text { for each } t \in\right] 0, \infty[.
$$

Indeed, $P_{x, x, y}(t)=\sup \{c \in[0,1]: P(x, x, t) * c=P(x, y, t)\}$, which is unique. Furthermore, as a consequence of the axiom (FPKM4) in Definition 3.1, we can deduce that, for each $x, y \in X$, the function $P_{x, x, y}$ is non-decreasing. However, such a function is not left-continuous in general, as shows the following example.

Example 4.2. Let $(X, P, \wedge)$ be the fuzzy partial metric space of Example 3.6. Consider $x=\frac{1}{4}$ and $y=\frac{1}{2}$. Then, $P(x, x, t)=\frac{t}{4(t+1)}$ for each $\left.t \in\right] 0, \infty[$ and

$$
P(x, y, t)= \begin{cases}\frac{t^{2}}{4(t+1)}, & \text { if } t \in] 0,1] ; \\ \frac{t}{4(t+1)}, & \text { elsewhere. }\end{cases}
$$

Therefore, attending to equation (3) the function $P_{x, x, y}$ is given by

$$
P_{x, x, y}(t)= \begin{cases}\frac{t^{2}}{4(t+1)}, & \text { if } t \in] 0,1[ \\ 1, & \text { if } t \in[1, \infty[\end{cases}
$$

which is not left-continuous at $t=1$.

In our next construction of a topology $\mathcal{T}_{P}$ deduced from $P$ we shall need leftcontinuity for $P_{x, x, y}$. To overcome this lack of left-continuity of the function $P_{x, x, y}$, in general, we propose to define the open balls in a fuzzy partial metric space as follows.

Definition 4.3. Let $(X, P, *)$ be a fuzzy partial metric space. We define the open ball $B_{P}$ centred at $x \in X$, with radius $\left.r \in\right] 0,1[$ and parameter $t \in] 0, \infty[$ by the next expression:

$$
B_{P}(x, r, t)=\left\{y \in X: P_{x, x, y}^{\prime}(t)>1-r\right\},
$$

where $P_{x, x, y}^{\prime}(t)=\sup \left\{P(x, x, s) \rightarrow_{*} P(x, y, s): s \in\right] 0, t[\}$.

Attending to the above observations on the assignment $P_{x, x, y}(t)$, it is not hard to check that $P_{x, x, y}^{\prime}$ is a non-decreasing function on $] 0, \infty[$, for each $x, y \in X$. Furthermore, $P_{x, x, y}^{\prime}$ is left-continuous, for each $x, y \in X$, as shows the next lemma.

Lemma 4.4. Let $(X, P, *)$ be a fuzzy partial metric space. For each $x, y \in X$, the function $\left.P_{x, x, y}^{\prime}:\right] 0, \infty[\rightarrow[0,1]$ given by

$$
\left.P_{x, x, y}^{\prime}(t)=\sup \left\{P(x, x, s) \rightarrow_{*} P(x, y, s): s \in\right] 0, t[\}, \text { for each } t \in\right] 0, \infty[,
$$

is left-continuous.

Proof. Take $x, y \in X$. We define, for each $t \in] 0, \infty[$, the function

$$
P_{x, x, y}^{\prime}(t)=\sup \left\{P(x, x, s) \rightarrow_{*} P(x, y, s): s \in\right] 0, t[\} .
$$


Let $\left.t_{0} \in\right] 0, \infty\left[\right.$. Since $\left(P\left(x, x, s_{2}\right) \rightarrow_{*} P\left(x, y, s_{2}\right)\right) \geq\left(P\left(x, x, s_{1}\right) \rightarrow_{*} P\left(x, y, s_{1}\right)\right)$, whenever $\left.s_{1}, s_{2} \in\right] 0, \infty$ [ with $s_{2}>s_{1}$, and attending to the definition of $P_{x, x, y}^{\prime}$, we deduce that for each $\epsilon>0$ there exists $\left.s_{0} \in\right] 0, t_{0}[$ such that

$$
P\left(x, x, s_{0}\right) \rightarrow_{*} P\left(x, y, s_{0}\right)>P_{x, x, y}^{\prime}\left(t_{0}\right)-\epsilon .
$$

Then, by the last inequality, we have that

$$
P(x, x, s) \rightarrow_{*} P(x, y, s)>P_{x, x, y}^{\prime}\left(t_{0}\right)-\epsilon, \text { for each } s \in\left[s_{0}, t_{0}\right] .
$$

Therefore, given $\epsilon>0$ there exists $\delta>0$, with $\delta=t_{0}-s_{0}$, such that

$$
P_{x, x, y}^{\prime}(t)=\sup \left\{P(x, x, s) \rightarrow_{*} P(x, y, s): s \in\right] 0, t[\}>P_{x, x, y}^{\prime}\left(t_{0}\right)-\varepsilon,
$$

for each $\left.t \in] t_{0}-\delta, t_{0}\right]$. Thus, $P_{x, x, y}^{\prime}$ is left continuous at $t_{0}$, and since $t_{0}$ is arbitrary then $P_{x, x, y}^{\prime}$ is left-continuous on $] 0, \infty[$.

Remark 7. Observe that if, for each $x, y \in X$, the function $P_{x, x, y}$ is left-continuous, then $\tilde{B}_{P}(x, r, t)=B_{P}(x, r, t)$, for each $\left.x \in X, r \in\right] 0,1[$ and $t \in] 0, \infty[$.

Lemma 4.5. Let $(X, P, *)$ be a fuzzy partial metric space. Then, for each $x, y, z \in X$ and each $t, s \in] 0, \infty[$ it is satisfied the following:

$$
P_{x, x, z}^{\prime}(t+s) \geq P_{x, x, y}^{\prime}(t) * P_{y, y, z}^{\prime}(s) .
$$

Proof. Let $x, y, z \in X$ and $t, s \in] 0, \infty\left[\right.$. Consider $\left.t^{\prime} \in\right] 0, t\left[\right.$ and $s^{\prime} \in$ ] $0, s\left[\right.$. Then, $P_{x, x, z}^{\prime}(t+s) \geq P\left(x, x, t^{\prime}+s^{\prime}\right) \rightarrow_{*} P\left(x, z, t^{\prime}+s^{\prime}\right) \geq$ $\left(P\left(x, x, t^{\prime}\right) \rightarrow_{*} P\left(x, y, t^{\prime}\right)\right) *\left(P\left(y, y, s^{\prime}\right) \rightarrow_{*} P\left(y, z, s^{\prime}\right)\right)$. Since $\left.t^{\prime} \in\right] 0, t\left[\right.$ and $s^{\prime} \in$ ] $0, s\left[\right.$ are arbitrary then $P_{x, x, z}^{\prime}(t+s) \geq\left(\sup \left\{P\left(x, x, t^{\prime}\right) \rightarrow_{*} P\left(x, y, t^{\prime}\right): t^{\prime} \in\right] 0, t[\}\right) *$ $\left(\sup \left\{P\left(y, y, s^{\prime}\right) \rightarrow_{*} P\left(y, z, s^{\prime}\right): s^{\prime} \in\right] 0, s[\}\right)=P_{x, x, y}^{\prime}(t) * P^{\prime}(y, y, z)(s)$.

Now, we are able to show the main goal of this section. We have included the proof for the sake of completeness and because of its particularities.

Theorem 4.6. Let $(X, P, *)$ be a fuzzy partial metric space. Then, the family of open balls

$$
\mathcal{B}=\left\{B_{P}(x, r, t): x \in X, r \in\right] 0,1[, t \in] 0, \infty[\}
$$

forms a base of a topology $\mathcal{T}_{P}$, which is $T_{0}$.

Proof. Let $\left.x_{1}, x_{2} \in X, r_{1}, r_{2} \in\right] 0,1\left[, t_{1}, t_{2} \in\right] 0, \infty[$ and consider the open balls $B_{P}\left(x_{1}, r_{1}, t_{1}\right)$ and $B_{P}\left(x_{2}, r_{2}, t_{2}\right)$.

We will show that for each $y \in B_{P}\left(x_{1}, r_{1}, t_{1}\right) \cap B_{P}\left(x_{2}, r_{2}, t_{2}\right)$ we can find $\left.r \in\right] 0,1[$ and $t \in] 0, \infty$ [ satisfying $B_{P}(y, r, t) \subset B_{P}\left(x_{1}, r_{1}, t_{1}\right) \cap B_{P}\left(x_{2}, r_{2}, t_{2}\right)$.

Let $y \in B_{P}\left(x_{1}, r_{1}, t_{1}\right) \cap B_{P}\left(x_{2}, r_{2}, t_{2}\right)$. Then, by definition of open ball, we have that

$$
P_{x_{1}, x_{1}, y}^{\prime}\left(t_{1}\right)>1-r_{1} \quad \text { and } \quad P_{x_{2}, x_{2}, y}^{\prime}\left(t_{2}\right)>1-r_{2} .
$$


On the one hand, we can find $\left.r_{1}^{\prime} \in\right] 0, r_{1}\left[\right.$ and $\left.r_{2}^{\prime} \in\right] 0, r_{2}[$ such that

$$
P_{x_{1}, x_{1}, y}^{\prime}\left(t_{1}\right)>\left(1-r_{1}^{\prime}\right) \quad \text { and } \quad P_{x_{2}, x_{2}, y}^{\prime}\left(t_{2}\right)>\left(1-r_{2}^{\prime}\right) \text {. }
$$

And, on the other hand, since the functions $P_{x_{1}, x_{1}, y}^{\prime}$ and $P_{x_{2}, x_{2}, y}^{\prime}$ are non-decreasing left-continuous functions on $] 0, \infty\left[\right.$, then we can find $\left.t_{1}^{\prime} \in\right] 0, t_{1}\left[\right.$ and $\left.t_{2}^{\prime} \in\right] 0, t_{2}$ [ satisfying

$$
P_{x_{1}, x_{1}, y}^{\prime}\left(t_{1}^{\prime}\right)>\left(1-r_{1}^{\prime}\right) \quad \text { and } \quad P_{x_{2}, x_{2}, y}^{\prime}\left(t_{2}^{\prime}\right)>\left(1-r_{2}^{\prime}\right)
$$

Now, since $*$ is continuous, there exist $\left.r_{1}^{\prime \prime} \in\right] 0,1\left[\right.$ and $\left.r_{2}^{\prime \prime} \in\right] 0,1\left[\right.$ such that $\left(1-r_{1}^{\prime}\right) *$ $\left(1-r_{1}^{\prime \prime}\right)>\left(1-r_{1}\right)$ and $\left(1-r_{2}^{\prime}\right) *\left(1-r_{2}^{\prime \prime}\right)>1-r_{2}$, respectively.

Take $r=\min \left\{r_{1}^{\prime \prime}, r_{2}^{\prime \prime}\right\}$ and $t=\min \left\{t_{1}-t_{1}^{\prime}, t_{2}-t_{2}^{\prime}\right\}$. We will see that $B_{P}(y, r, t) \subset$ $B_{P}\left(x_{1}, r_{1}, t_{1}\right) \cap B_{P}\left(x_{2}, r_{2}, t_{2}\right)$. Indeed, take $z \in B_{P}(y, r, t)$, then applying Lemma 4.5 in the first inequality and the monotony of the function $P_{x_{1}, x_{1}, y}^{\prime}$ in the second one, we have that

$$
\begin{aligned}
P_{x_{1}, x_{1}, z}^{\prime}\left(t_{1}\right) & \geq\left(P_{x_{1}, x_{1}, y}^{\prime}\left(t_{1}^{\prime}\right)\right) *\left(P_{y, y, z}^{\prime}\left(t_{1}-t_{1}^{\prime}\right)\right) \geq\left(P_{x_{1}, x_{1}, y}^{\prime}\left(t_{1}^{\prime}\right)\right) *\left(P_{y, y, z}^{\prime}(t)\right) \geq \\
& \geq\left(1-r_{1}^{\prime}\right) *(1-r) \geq\left(1-r_{1}^{\prime}\right) *\left(1-r_{1}^{\prime \prime}\right)>\left(1-r_{1}\right),
\end{aligned}
$$

and so $z \in B_{P}\left(x_{1}, r_{1}, t_{1}\right)$. $\mathcal{T}_{P}$.

Analogously, one can prove that $z \in B_{P}\left(x_{2}, r_{2}, t_{2}\right)$. Hence, $\mathcal{B}$ is a base of a topology

Finally, we will prove that $\left(X, \mathcal{T}_{P}\right)$ is a $T_{0}$-space. Indeed, if $x \neq y$, there exists $\left.t_{0} \in\right] 0, \infty\left[\right.$ such that $P_{x, x, y}^{\prime}\left(t_{0}\right)<1$ or $P_{y, y, x}^{\prime}\left(t_{0}\right)<1$. Contrary, $P_{x, x, y}^{\prime}(t)=P_{x, x, y}^{\prime}(t)=1$, for each $t \in] 0, \infty$ [, that implies $P(x, x, t)=P(x, y, t)=P(y, y, t)$, for each $t \in] 0, \infty[$, which is equivalent to $x=y$. Suppose that $P_{x, x, y}^{\prime}\left(t_{0}\right)=1-r_{0}<1$. Let $r_{1}<r_{0}<1$. Then $y \notin B_{P}\left(x, r_{1}, t_{0}\right)$, and so $\left(X, \mathcal{T}_{P}\right)$ is a $T_{0}$-space.

Concerning the above theorem, given a fuzzy partial metric $P$ on a non-empty set $X$, we will say that $\mathcal{T}_{P}$ is the topology deduced from $P$ or induced by $P$.

Coming back to the fuzzy partial metric case, as it has been shown in Example 4.2, axioms (FPKM1)-(PKM5) do not ensure the left-continuity of the function $P_{x, x, y}$. Nevertheless, in such an example, one can verify that we are able to define a topology on $X$ deduced from $P$, which has as a base the family of open balls $\tilde{B}_{P}$. It is due to the fact that the fuzzy partial metric $P$, defined in Example 3.6, satisfies in addition the next condition, for each $x, y, z \in X$ and each $t \in] 0, \infty[$,

$$
P(x, x, t) \rightarrow_{*} P(x, z, t) \geq\left(P(x, x, t) \rightarrow_{*} P(x, y, t)\right) *\left(P(y, y, t) \rightarrow_{*} P(y, z, t)\right) .
$$

So, the above comments motivate the next question.

Question 4.7. Let $(X, P, *)$ be a fuzzy metric space. Can we define a topology on $X$ which has as a base the family

$$
\left\{\tilde{B}_{P}(x, r, t): x \in X, r \in\right] 0,1[, t \in] 0, \infty[\} ?
$$


Remark 8. Example 4.2 shows a fuzzy partial metric such that $P_{x, x, y}$ is not a leftcontinuous function but the family

$$
\left\{\tilde{B}_{P}(x, r, t): x \in X, r \in\right] 0,1[, t \in] 0, \infty[\}
$$

defines a topology on $X$.

\section{Conclusions}

In this paper we have introduced a notion of fuzzy partial metric space $(X, P, *)$ which constitutes an addaptation of the notion of partial metric space given in Matthews (1994) to the fuzzy setting. Such an addaptation is based on the fuzzy metric sapce given in Kramosil and Michalek (1975), using the residuum operator $\rightarrow_{*}$ associated to a continuous $t$-norm $*$, without any extra condition on $*$. Then, we have constructed a topology $\mathcal{T}_{P}$ on $X$, deduced from $P$, and we have proved that $\left(X, \mathcal{T}_{P}\right)$ is a $T_{0}$-space.

The introduced notion of fuzzy partial metric opens several lines of research in the fuzzy setting. On the one hand, the study of the (fuzzy partial) metric properties, and in particular fixed point theory. On the other hand, its applicability to engineering problems in which it makes sense to consider that the self-similarity can be less than 1.

\section{Acknowledgement}

The authors are very grateful to the reviewers for their valuable suggestions which have helped to improve the content of the paper.

\section{Funding}

Valentín Gregori acknowledges the support of the Ministry of Economy and Competitiveness of Spain under Grant MTM2015-64373-P (MINECO/Feder, UE).

Juan José Miñana acknowledges the partially support of the Spanish Ministry of Economy and Competitiveness under Grant TIN2016-81731-REDT (LODISCO II) and AEI/FEDER, UE funds, by the Programa Operatiu FEDER 2014-2020 de les Illes Balears, by project ref. PROCOE/4/2017 (Direcció General d'Innovació i Recerca, Govern de les Illes Balears), and by project ROBINS. The latter has received research funding from the EU H2020 framework under GA 779776. This publication reflects only the authors views and the European Union is not liable for any use that may be made of the information contained therein.

\section{References}

Birkhoff G. 1973. Lattice Theory. American Mathematical Society, Providence.

Bukatin M., Kopperman R., and Matthews S. 2014. "Some corollaries of the correspondence between partial metrics and multivalued equalities." Fuzzy Sets and Systems 256 57-72. 
Camarena J.G., Gregori V., Morillas S., and Sapena A. 2010. "Two-step fuzzy logic-based method for impulse noise detection in colour images.", Pattern Recognition Letters 31 (13) $1842-1849$.

Demirci M. 2012. "The order-theoretic duality and relations between partial metrics and local equalities." Fuzzy Sets and Systems 192 45-57.

George A., and Veeramani P. 1994. "On some results in fuzzy metric spaces." Fuzzy Sets and Systems 64 (3) 395-399.

George A., and Veeramani P. 1995. "Some theorems in fuzzy metric spaces." The Journal of Fuzzy Mathematics 3 933-940.

Grabiec M. 1988. "Fixed points in fuzzy metric spaces." Fuzzy Sets and Systems 27 (3) 385389.

Grečova S., and Morillas S. 2016. "Perceptual similarity between color images using fuzzy metrics." Journal of Visual Communication and Image Representation 34 230-235.

Gregori V., Miñana J.J., and Morillas S. 2012. "Some questions in fuzzy metric spaces." Fuzzy Sets and Systems 204 71-85.

Gregori V., Morillas S., and Sapena A. 2010. "On a class of completable fuzzy metric spaces." Fuzzy Sets and Systems 161 (16) 2193-2205.

Gregori V., and Romaguera S. 2000. "Some properties of fuzzy metric spaces." Fuzzy Sets and Systems 115 (3) 485-489.

Gregori V., and Sapena A. 2002. "On fixed-point theorems in fuzzy metric spaces." Fuzzy Sets and Systems 125 (2) 245-252.

Gutiérrez-Gracía J., and Rodríguez-López J., and Romaguera S. 2018. "On fuzzy uniformities induced by a fuzzy metric space." Fuzzy Sets and Systems 330 (1) 52-78.

Höhle U., and Klement E.P. 1995. Non-Classical Logics and Their Applications to Fuzzy Subsets. A Handbook of the Mathematical Foundations of Fuzzy Set Theory. Kluwer Academic Publishers, Dordrecht.

Klement E.P., Mesiar R., and Pap E. 2000. Triangular norms. Springer Netherlands.

Kramoosil I., and Michalek J. 1975. "Fuzzy metric and statistical metric spaces." Kybernetika $11336-344$.

Matthews S.G. 1994. "Partial Metric Topology." Annals of the New York Academy of Sciences $728183-197$.

Menger K. 1942. "Statistical metrics." Proceedings of the National Academy of Sciences of the United States of America 28 (12) 535-537.

Mihet D. 2008. "Fuzzy $\Psi$-contractive mappings in non-Archimedean fuzzy metric spaces." Fuzzy Sets and Systems 159 (6) 739-744.

Miñana J.J., and Valero O. 2018. "A duality relationship between fuzzy metrics and metrics." International Journal of Fuzzy Systems 47 (6) 593-612

Schweizer B., and Sklar A. 1960. "Statistical metric spaces." Pacific Journal of Mathematics $10314-334$.

Sedghi S., Shobkolaei N., and Altun I. 2015. "Partial fuzzy metric space and some fixed point results." Communications in Mathematics 23 131-142.

Shukla S., Gopal D., and Roldán-López-de-Hierro A.F. 2016. "Some fixed point theorems in 1-M-complete fuzzy metric-like spaces." International Journal of General Systems 45 (7-8) $815-829$.

Wu J., and Yue Y. 2017. "Formal balls in fuzzy partial metric spaces." Iranian Journal of Fuzzy Systems 14 (2) 155-164.

Ying M. 1991. "A new approach for fuzzy topology (I)." Fuzzy Sets and Systems 39 303-321. Yue Y., and Gu M. 2014. "Fuzzy partial (pseudo-)metric spaces." Journal of Intelligent and Fuzzy Systems 27 1153-1159.

Yue Y. 2015. "Separated $\Delta^{+}$-valued equivalences as probabilistic partial metric spaces." Journal of Intelligent and Fuzzy Systems 28 2715-2724. 